\title{
Monocyte Derived Interleukin (IL)-23 Is an Important Determinant of Synovial IL-17A Expression in Rheumatoid Arthritis
}

\author{
LISA K. STAMP, ANDREA EASSON, LINDA PETTERSSON, JOHN HIGHTON, and PAUL A. HESSIAN
}

\begin{abstract}
Objective. To demonstrate gene expression of interleukin (IL)-17A, IL-23, and IL-12 and to determine the proximity of IL-17A and IL-23 producing cells in rheumatoid synovial tissue.

Methods. Total RNA was isolated from 25 synovial membranes obtained from 20 patients with rheumatoid arthritis (RA). Quantitative real-time polymerase chain reaction was used to measure IL-17A, IL-12p35, IL-23p19, p40, and GAPDH expression. Immunohistochemistry was utilized to determine cell type and proximity of IL-17A, IL-12, and IL-23 in rheumatoid synovium.

Results. IL-17A was present in 13/25 synovia. IL-12p35 was present in all samples while IL-23p19 was present in 23/25. p40 was present in 23/25 samples. Of the 2 p40- samples both were IL-23p19 and IL-12p35 positive. Mean expression of IL-23p19 was significantly higher in the IL-17A+ versus IL-17A- synovia $(0.10 \pm 0.02 \mathrm{ng}$ vs $0.05 \pm 0.01 \mathrm{ng} ; \mathrm{p}<0.05)$. There was no difference in IL-12p35 expression between IL-17A + and IL-17A- synovia $(0.5 \pm 0.21 \mathrm{ng}$ vs $0.38 \pm 0.24 \mathrm{ng} ; \mathrm{p}=$ $0.2)$. All IL-17A+ cells were in the vicinity of IL-23+ cells. IL-12+ cells were both close to and removed from IL-17A+ cells. Only a proportion of CD3+T cells appeared to produce IL-17A.

Conclusion. IL-17A gene expression occurs in only a subset of rheumatoid synovial membranes. IL-23 gene expression is higher in IL-17A+ versus IL-17A- membranes. In keeping with this, IL-17A + and IL-23+ cells colocalize in synovial membranes. IL-17 is not an absolute requirement in RA but may be important in amplifying the inflammatory response. Anti-IL23 therapies may have a role in those patients with IL-17A expression. (First Release Oct 1 2009; J Rheumatol 2009;36:2403-8; doi:10.3899/jrheum.081304)
\end{abstract}

Key Indexing Terms:

RHEUMATOID ARTHRITIS INTERLEUKIN-17A INTERLEUKIN-23 INTERLEUKIN-12

Rheumatoid arthritis (RA) is a heterogeneous disease ranging from mild inflammation with little joint damage to a crippling arthritis with severe joint destruction. The primary lesion in RA occurs in the synovium, which contains inflammatory cells including monocytes/macrophages, $\mathrm{B}$, and $\mathrm{T}$ cells. These cells produce an array of proinflammatory cytokines and proteolytic enzymes that ultimately lead to bone and cartilage damage and joint destruction.

From the Department of Medicine, University of Otago, Christchurch; Leukocyte Inflammation Research Laboratory, Department of Physiology, University of Otago; and Department of Medicine and Surgical Sciences, University of Otago, Dunedin, New Zealand.

Supported by Lotteries Health New Zealand and New Zealand Health Research Council.

L.K. Stamp, PhD, FRACP, Department of Medicine, University of Otago, Christchurch; A. Easson, MD; L. Pettersson, MD, Leukocyte

Inflammation Research Laboratory, Department of Physiology, University of Otago; J. Highton, MD, FRACP, Department of Medicine and Surgical Sciences, University of Otago, Dunedin; P.A. Hessian, PhD, Leukocyte Inflammation Research Laboratory, Department of Physiology, University of Otago.

Address correspondence to Dr. L.K. Stamp, Department of Medicine, University of Otago, Christchurch, P.O. Box 4345, Christchurch,

New Zealand.E-mail: lisa.stamp@cdhb.govt.nz

Accepted for publication June 26, 2009.
The interleukin (IL)-17 family is a family of cytokines composed of 6 members, IL-17A-F. Of these, IL-17A is the best described in association with RA and has been reported to be present in $28-80 \%$ of synovial membranes ${ }^{1,2}$. IL-17A has a number of proinflammatory actions both directly and through synergy with IL-1ß and tumor necrosis factor (TNF)- $\alpha$. These include stimulating production of IL-6, IL-8, IL-1ß, TNF- $\alpha$, and prostaglandin E2 by monocytes/ macrophages and synoviocytes, inducing expression of matrix metalloproteinases, stimulating osteoclast precursors, inhibiting proteoglycan synthesis, and increasing expression of receptor activator of nuclear factor- $\kappa \mathrm{B}$ (RANK) and RANK ligand (RANKL), thereby promoting osteoclast-mediated bone destruction. In murine models, inhibition of IL-17A with neutralizing antibodies has been shown to suppress the onset of experimentally induced arthritis, reduce the severity of the arthritis, and reduce synovial RANKL mRNA expression and bone erosion. Thus IL-17 appears to link inflammation and bone erosion in RA.

A specific subset of IL-17A- producing T helper cells (Th17 cells) has been identified. In murine models, development of Th17 cells is promoted through the action of IL-6 and transforming growth factor (TGF)- $\beta$ on naïve murine CD4+ T cells, while IL-23 is important in maintenance and

Personal non-commercial use only. The Journal of Rheumatology Copyright @ 2009 . All rights reserved. 
expansion of Th17 cells ${ }^{3}$. In contrast, interferon (IFN)- $\gamma$, IL-2, IL-4, IL-25, and IL-27 inhibit the development of murine Th17 cells ${ }^{4}$.

While the mechanisms leading to human Th17 cell development and IL-17A production have not been fully elucidated, it has become clear that the key human cytokines differ from those driving the murine IL-17 system. IL-12 and IL-23 are members of the IL-12 cytokine family. IL-23 consists of a unique p19 subunit and a p40 subunit shared with IL-12. IL-23 has an important role in Th17 cell differentiation and IL-17A production in humans ${ }^{5,6}$. In comparison, IL-12, which consists of a unique p35 subunit and the p40 subunit shared with IL-23, inhibits IL-17A production ${ }^{6}$. IL-1ß, IL-6, and TGF- $\beta$ have also been implicated in Th17 cell differentiation in humans ${ }^{7}$.

It remains unclear why some patients with RA develop more severe and more destructive disease compared to others. The ability to predict which patients will develop more severe disease at the time of diagnosis remains one of the current challenges in the management of RA. It has been reported that synovial membrane mRNA levels of IL-1ß, TNF- $\alpha$, IL-17A, and IL-10 are predictive of progressive joint damage over 2 years. Further, IL-17A and TNF- $\alpha$ were synergistic and the effects of these 2 cytokines were more pronounced with shorter disease duration ${ }^{1}$. Thus IL-17A appears to have an important proinflammatory and joint destructive effect in RA and may amplify the effects of TNF- $\alpha$.

The aim of our study was to measure IL-17A gene expression in synovial membranes from patients with RA, and to determine corresponding levels of IL-23 and IL-12 gene expression in synovial membranes with and without IL-17A. In addition we wished to identify those cells producing IL-17A and IL-23 and their relative proximity to each other within the synovial membrane.

\section{MATERIALS AND METHODS}

Ethical approval was obtained from the New Zealand Multiregional Ethics Committee. We obtained 25 synovial samples from 20 patients fulfilling American College of Rheumatology (ACR) criteria for RA undergoing joint surgery ${ }^{8}$.

Cytokine gene expression. At the time of surgery synovial samples were either placed in RNAlater (Ambion, Austin, TX, USA) for short-term storage, or divided and immediately snap frozen in liquid nitrogen for longterm storage. Total RNA was extracted from 50-100 mg of synovial tissue using Qiagen RNeasy mini kits (Qiagen, Hilden, Germany). Extracted RNA was stored at $-80^{\circ} \mathrm{C} \cdot 0.5-1 \mu \mathrm{g}$ of RNA was reverse transcribed at $42^{\circ} \mathrm{C}$ for 50 min using Superscript II (Life Technologies, Carlsbad, CA) and Oligo (dT) $)_{12-18}$ primers. Quantitative real-time polymerase chain reaction (PCR) was undertaken using Taqman gene expression assays (Applied Biosystems, Foster City, CA) for IL-17A, IL-12p35, IL-23p19, p40, and GAPDH. Analysis of each sample was undertaken in triplicate and results expressed as the mean $\mathrm{ng} \pm$ standard deviation $(\mathrm{SD})$ of the gene of interest relative to the mean ng of GAPDH RNA expression.

Immunohistochemistry. The presence of IL-17A, IL-23, or IL-12 protein was assessed using commercially available rabbit (anti-IL-23; AbD Serotec, Kidlington, UK) or goat (IL-17A and IL-12; R\&D Systems,
Minneapolis, MN, USA) antibodies optimally diluted in RPMI-1640 containing $10 \%$ heat inactivated fetal calf serum (FCS) and $100 \mathrm{ug} / \mathrm{ml}$ sheep (anti-IL-23) or rabbit (anti-IL-17A and anti-IL-12) immunoglobulins. Consecutive cryostat sections of synovial tissue were incubated with these primary antibodies for $3 \mathrm{~h}$, then washed with three 5-min incubations in phosphate buffered saline (PBS). Endogenous peroxidase activity was blocked with $30 \mathrm{~min}$ incubation in $0.3 \% \mathrm{H}_{2} \mathrm{O}_{2}$ followed by application of species-specific secondary antibodies [anti-rabbit Ig (Dako, Glostrup, Denmark) or anti-goat Igs] for $2 \mathrm{~h}$. Following a further wash sequence, bound antibody was detected using 3, 3'-diaminobenzidine (DAB) substrate. All sections were counterstained with hemotoxylin before mounting in DPX. To identify various cell types responsible for the production of IL-17A and IL-23, consecutive sections were stained with monoclonal antibodies recognizing either $\mathrm{T}$ cells (anti-CD3; Dako), monocyte/ macrophages (anti-CD14; clone FMC17, inhouse clone), dendritic cells (antibody CMRF44; courtesy of Derek Hart, Brisbane), or B cells (anti-CD20; Dako). Monoclonal antibodies were detected with rabbit anti-mouse Ig (Dako) and DAB substrate as described above.

Statistics. Values were compared using a Student's t test and correlations using a Spearman test.

\section{RESULTS}

Details of the patients are shown in Table 1. From the 20 patients, 9 synovia were IL-17A+, 10 were IL-17A-, and the remaining 2 synovia, from a single patient, were IL-17A+ and IL-17A-, respectively (Table 1).

IL-17A only expressed in a subset of rheumatoid synovial tissue samples. Of the 25 synovial samples, 13 (52\%) were positive for IL-17A gene expression. The mean $\pm \mathrm{SD}$ expression of IL-17A in the positive samples was $0.10 \pm$ $0.08 \mathrm{ng}$ (range 0.012-0.26 ng).

Five patients provided 2 synovial samples. Of these, 2 patients had samples from different sites obtained on the same day. In one case both samples were positive for IL-17A and in the other, both samples were IL-17A-. Two patients had serial synovial samples, obtained 5 years and 14 months apart; all were positive. In the final case, the initial sample was IL-17A- while the second sample obtained 5 months later was IL-17A+.

There was no significant correlation between the presence of synovial IL-17A and disease duration, erythrocyte sedimentation rate, or C-reactive protein (data not shown). In the case of the IL-17A+ samples 6/13 (46\%) patients were receiving therapy with corticosteroids compared to $6 / 12(50 \%)$ of the IL-17A- patients. Of the IL-17+ samples, $6 / 13(46 \%)$ patients were receiving MTX compared to 4/12 (33\%) patients with IL-17A-samples.

Expression of IL-12p35, IL-23p19 and p40 in rheumatoid synovium. IL-12p35 was expressed in all 25 synovial samples. Mean expression was $0.45 \pm 0.05 \mathrm{ng}$ (range $0.10-0.99$ ng). IL-23p19 was expressed in 23/25 (92\%) synovia. Mean expression in the IL-23p19 positive synovia was $0.08 \pm 0.06$ ng (range 0.007-0.245 ng). The common p40 subunit was expressed in 23/25 synovia. Mean expression in the positive synovia was $0.49 \pm 0.14 \mathrm{ng}(0.02-1.56 \mathrm{ng})$. Of the 2 synovia that did not express p40, both were IL-23p19 and IL-12p35 positive. However, one was IL-17A+ and one IL-17A-. 
Table 1. Characteristics of the 20 patients from whom rheumatoid synovial tissue was obtained. One patient had 2 biopsies of which 1 was IL-17A+ and 1 IL-17A-. This patient was excluded from the analysis of IL-17A+ vs IL17A- in the table.

\begin{tabular}{lccc}
\hline & All Patients, & IL-17A+, & IL-17A-, \\
& $\mathrm{n}=20$ & $\mathrm{n}=9$ & 10 \\
\hline Mean age, yrs (range) & $60.7(35-80)$ & $59.6(35-74)$ & $61.5(36-80)$ \\
Male:female & $6: 14$ & $3: 6$ & $2: 8$ \\
RF positive, $\mathrm{n}(\%)$ & $18(90)$ & $8(88.9)$ & $9(90)$ \\
Radiographic erosions, $\mathrm{n}(\%)$ & $19(95)$ & $8(88.9)$ & $10(100)$ \\
Disease duration, yrs, mean (range) & $16.9(2-30)$ & $16.1(5-29)$ & $19.2(8-30)$ \\
ESR, mm/h, mean (range) & $30(0-85)$ & $21(0-31)$ & $39(18-85)$ \\
CRP, mg/l, mean (range) & $11(0-70)$ & $6.7(0-26)$ & $8(0-38)$ \\
DMARD, $\mathrm{n}(\%)$ & $18(90)$ & $8(88.9)$ & $8(80)$ \\
Methotrexate, $\mathrm{n}(\%)$ & $10(50)$ & $5(55.5)$ & $4(40)$ \\
Methotrexate dose mg/wk, mean (range) & $16(10-22.5)$ & $18(15-22.5)$ & $14.1(10-17.5)$ \\
Prednisone & 12 & 5 & 6 \\
Prednisone dose mg/day, mean (range) & $6(4-10)$ & $6.5(4-10)$ & $6(5-10)$ \\
\hline
\end{tabular}

RF: rheumatoid factor; ESR: erythrocyte sedimentation rate; CRP: C-reactive protein; DMARD: disease modifying antirheumatic drugs.

Relationships between IL-23p19, IL-12p35, p40, and $I L-17 A$. There was no difference in mean IL-12p35 expression between IL-17A+ and IL-17A- synovia $(0.5 \pm 0.06 \mathrm{ng}$ vs $0.38 \pm 0.07 \mathrm{ng} ; \mathrm{p}=0.2)$. There was no significant correlation between IL-12p35 and IL-17A expression (Spearman $r=0.37, p=0.07)$. In all synovia the expression of IL-12p35 was higher than that of IL-23p19 (mean expression $0.45 \pm$ 0.02 vs $0.07 \pm 0.01 ; \mathrm{p}<0.0001)$.

The expression of IL-23p19 was significantly higher in IL-17A positive synovia as compared to those that were IL-17A negative $(0.10 \pm 0.02 \mathrm{ng}$ vs $0.05 \pm 0.01 \mathrm{ng} ; \mathrm{p}=0.02)$ (Figure 1). Interestingly, there was 1 synovium positive for IL-17A where IL-23p19 could not be detected. There was a significant positive correlation between IL-23p19 expression and IL-17A expression (Spearman $r=0.44, p=0.03$ ). There was also a significant positive correlation between the

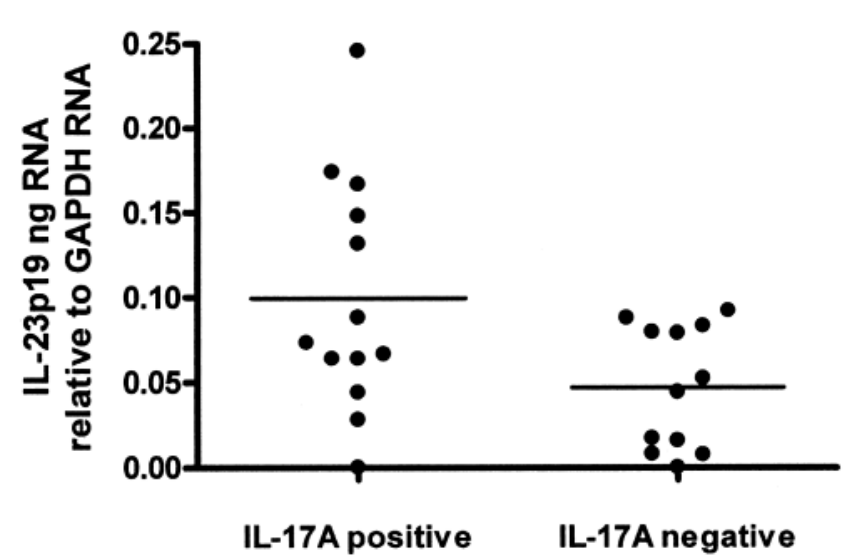

Figure 1. Quantitative RNA expression of IL-23p19 in IL-17A positive and IL-17A negative synovial membrane samples. Each point represents data from an individual synovial sample, with the bar representing the mean of the group $(\mathrm{p}=0.02)$. ratio of IL-23p19/IL-12p35 and IL-17A expression (Spearman's $r=0.43, p=0.02$ ) (Figure 2).

There was no difference in mean expression of $\mathrm{p} 40$ in IL- $17 \mathrm{~A}+$ and IL- $17 \mathrm{~A}-$ samples $(0.53 \pm 0.13$ vs $0.37 \pm 0.09$; $\mathrm{p}=0.33)$. There was no correlation between $\mathrm{p} 40$ and expression of IL-17A ( $=0.39)$, IL-23p19 ( $\mathrm{p}=0.13)$, or IL-12p35 $(\mathrm{p}=0.12)$. There was significantly more expression of $\mathrm{p} 40$ than IL-23p19 (0.45 \pm 0.08 vs $0.07 \pm 0.01 ; \mathrm{p}<0.0001)$, but no difference between $\mathrm{p} 40$ and IL-12p35 (p = 0.96). In $13 / 25$ samples there was excess $\mathrm{p} 40$ over the combination of IL-23p19 and IL-12p40. Four of 13 IL-17A+ samples and 4/12 IL-17A- samples had an excess of p40 over the combination of IL-12p35 and IL-23p19.

Proximity of cells producing $I L-17 A, I L-23$, and $I L-12$ in rheumatoid synovium. Immunohistochemistry revealed that only a very small number of CD3 $+\mathrm{T}$ cells were also posi-

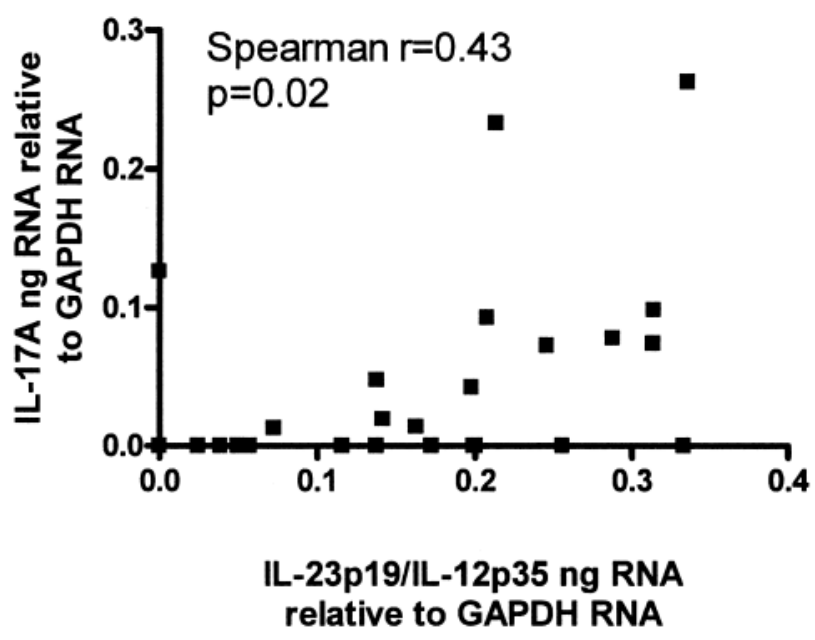

Figure 2. Correlation of IL-23p19/IL-12p35 ratio to IL-17A expression. Each point represents data from an individual synovial membrane sample.

Personal non-commercial use only. The Journal of Rheumatology Copyright @ 2009. All rights reserved. 
tive for IL-17A. We found no evidence for IL-17A production by any other cell types. IL-23 staining was present throughout the synovial membrane. IL-17A+ cells were in close proximity to different cells staining strongly for IL-23+. There was no evidence of IL-17A in the absence of IL-23. Further, there was no relationship between the distribution of IL-17A+ cells and IL-12+ cells. CMRF44+ dendritic cells were negative for IL-23 while FMC17+ monocytes were IL-23+ (Figure 3).

\section{DISCUSSION}

IL-17A is expressed in only a subset of rheumatoid synovial membranes. Thus IL-17A is not an absolute requirement for inflammation in RA. Rather, current evidence would suggest that it has an important role in amplifying disease in the subset of patients in whom it is present. In this regard, synovial membrane IL-17A mRNA levels have been reported to be predictive of joint damage in patients with $\mathrm{RA}^{1}$. Accurate prediction of those patients with poorer prognosis and making an early decision on which of the multiple treatments available is most likely to rapidly and effectively suppress inflammatory disease activity remain key challenges in the current management of patients with RA. The expression of IL-17A in approximately half of RA synovial samples and the association between IL-17A and joint damage provides one mechanism whereby patients with RA may be classified with regard to prognosis. It is relevant in this context to note that IL-17A cannot be detected in rheumatoid nodules, which are found in that group of patients with more severe disease and increased mortality ${ }^{9}$. Whether patients with synovial IL-17A expression are more or less likely to respond to specific therapies remains to be determined.

Reported expression of IL-17A in rheumatoid synovial samples has ranged from $28 \%$ to $80 \%$ of samples ${ }^{1,2}$. Further, IL-17A has been detected in $\sim 45 \%$ of rheumatoid synovial fluid samples ${ }^{10}$. Although our finding of IL-17A in 50\% of samples is higher than the $28 \%$ positive samples reported by Kirkham, et al, there are significant differences between the 2 populations in these studies. Most notably, our samples were obtained from patients undergoing joint surgery from a variety of sites, compared to Kirkham, et al, where all samples were obtained from knee joints not at the time of surgery. In addition, Kirkham, et al had a significant number of patients with disease duration $<2$ years.

Synovial expression of IL-17A is not unique to RA and it is also detectable in synovium from patients with spondyloarthropathies and osteoarthritis. Interestingly, IL-17A concentrations are significantly higher in synovial fluid and synovial tissues from patients with spondyloarthropathies compared to RA ${ }^{11}$. While IL-17A is clearly a proinflammatory cytokine, it is present in only small numbers of patients with osteoarthritis ${ }^{2,10,11}$.

While the exact mechanisms of Th17 cell differentiation and IL-17A production remain to be clarified in humans it is apparent that both IL-23 and IL-12 have some role. IL-23 is a heterodimeric cytokine consisting of a unique p19 subunit and a p40 subunit that is shared with IL-12. IL-23p19 is upregulated in rheumatoid synovial fibroblasts with expres-

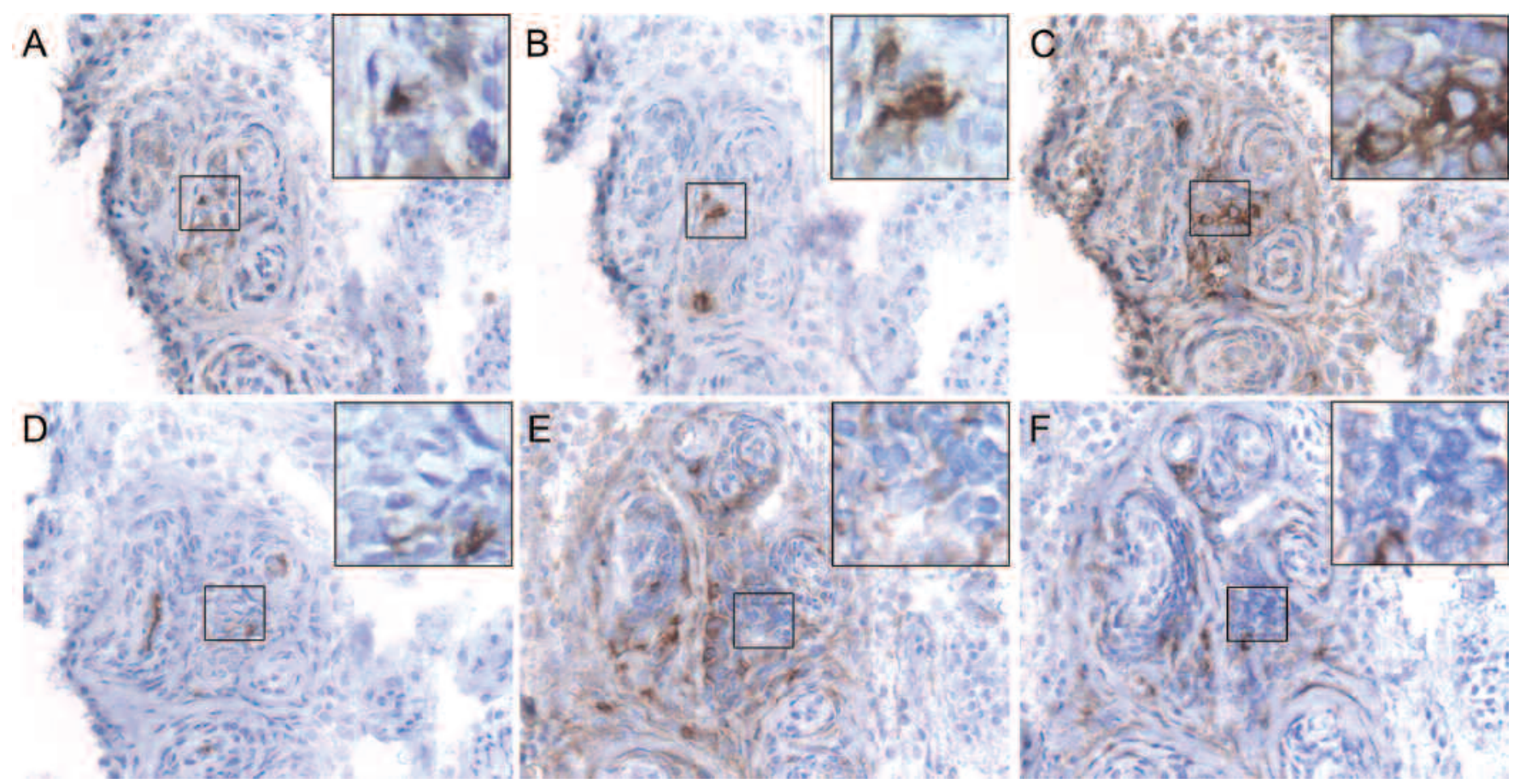

Figure 3. Immunohistochemistry images at low power magnification $(20 \times)$ and high power insert (63×). A. T cell marker CD3, B. IL-17A, C. IL-23, D. IL-12, E. Monocyte/macrophage marker FMC17, F. Dendritic cell marker CMRF44.

Personal non-commercial use only. The Journal of Rheumatology Copyright @ 2009 . All rights reserved. 
sion positively controlled by the presence of IL-17 $\mathrm{A}^{12}$. IL-23 also induces Th17 cell differentiation and IL-17A production ${ }^{5,6}$. Thus there appears to be an important positive feedback loop between IL-23 and IL-17A, which may be important in driving synovial inflammation. We have shown that IL-23p19 gene expression is significantly higher in IL-17A+ compared to IL-17A- synovial membranes. Further, there was a positive correlation between the ratio of IL-23p19/IL-12p35 expression and IL-17A expression. A similar correlation between IL-23p19 and IL-17A protein concentrations in synovial fluid and sera from patients with RA has been reported ${ }^{13,14}$. In addition we have shown close proximity between IL-17A+ and IL-23+ cells. These data give additional support to the role of IL-23 in IL-17A production in rheumatoid synovial inflammation.

Biologically active IL-23 and IL-12 require synthesis of the 2 subunits, p19 and p40 for IL-23 and p35 and p40 in the case of IL-12, by the same cell ${ }^{15}$. It has recently been reported that while IL-23p19 is expressed in rheumatoid synovial tissue, few cells co-express the p19 and p40 subunits. Moreover, IL-23 was present in only 6/37 RA synovial fluid samples ${ }^{16}$. Accordingly, the authors suggest that the presence of p19 does not necessarily indicate the presence of a bioactive IL-23 heterodimer ${ }^{16}$. We have shown that the majority of synovia express p 40 and that there is generally an excess of p40 over p19 and p35. This would suggest that dissociation between p19 and p40 is unlikely to have a substantial influence on our results. Further, there was no difference between the numbers of patients with an excess of p40 in the IL-17A+ and IL-17A- samples. However, it is expression of the IL-23p19 subunit by monocyte-derived dendritic cells that is of relevance. These cells also produce IL-12p35 and enough IL-12p40 subunit to satisfy requirements for both IL-12 and IL-23 heterodimers. Thus production of IL-23p19 by myeloid dendritic cells may be the rate-limiting step in IL-23 heterodimer expression. Interestingly, we observed that IL-23+ cells were FMC17+ monocytes, while CMRF44+ dendritic cells were IL-23-.

Interestingly, in 1 patient IL-17A gene expression was present despite the absence of IL-23. Serum concentrations of IL-23 have been reported to decrease significantly after treatment with etanercept. However, there was no associated change in IL-17A concentrations ${ }^{14}$. These data suggest that other as yet unidentified factors may be important in development and/or maintenance of Th17 cells and IL-17A production in humans. Alternatively, medications used for disease suppression may alter Th17 cell development and IL-17A production. It has been suggested in patients with giant cell arteritis that circulating Th17 cells markedly decrease with steroid therapy ${ }^{17}$. However, in our IL-17A+ samples $50 \%$ of patients were receiving steroids at the time of sample collection.

Notwithstanding the presence of IL-17A in RA synovium, only $\sim 1 \%$ of $\mathrm{T}$ cells present within the rheumatoid joint have been reported to produce IL- $17 \mathrm{~A}^{2}$. More recently, IL-17A+CD4+ T cells from rheumatoid synovium or synovial fluid have been reported to be scarce, with IL-17A+ T cells found less frequently in joints compared to peripheral blood mononuclear cells ${ }^{18}$. One explanation for the paucity of IL-17A+ T cells is that IL-17A is expressed only in certain phases of the $\mathrm{T}$ cell activation process. The murine system suggests IL-17A production by T cells is suppressed by IL-27 when CD4+ T cells are in the early stages of $\mathrm{T}$ cell activation but not when they are fully activated ${ }^{19}$. Similarly, suppression of Th17 cell development by IFN- $\gamma$ and IL-4 appears to be limited to an early stage of Th17 differentiation ${ }^{4}$.

An alternative explanation is that IL-17A is only present in certain phases of the disease process. In humans, IL-17A has been reported to be higher in synovial fluid from patients with early RA as compared to those with established disease ${ }^{20}$. However, in our cohort of patients with longstanding disease ( $>2$ years) we found no association between IL-17A expression and disease duration. Interestingly, in our cohort there was 1 patient in whom 2 samples were obtained 5 months apart. In the initial sample IL-17A was not present, whereas in the subsequent sample IL-17A was present. This patient had the shortest disease duration (2 years) of all the patients in the study. Whether the change in IL-17A status in this patient is due to different phases of $\mathrm{T}$ cell activation or different phases of the disease is unclear. While these data suggest that the presence/ absence of IL-17A can vary within an individual patient (i.e., negative to positive), we have found no evidence for reversion of the IL-17A positive synovial status to negative.

In summary, IL-17A is expressed in only a proportion of rheumatoid synovial membrane samples. Thus, IL-17A is not an absolute requirement for RA. Rather it may be an important amplifier of inflammatory disease activity. The association of IL-17A with poorer disease outcomes provides a rationale for identification of that subset of patients with synovial expression of IL-17A. IL-23 is an inducer of IL-17A production, and expression of IL-23p19 is higher in IL-17A+ membranes as compared to IL-17A- membranes. Given the links between IL-23 and IL-17, therapeutic agents specifically targeting these cytokines in that subgroup of patients who express IL-17A may be of benefit.

\section{ACKNOWLEDGMENT}

We are grateful to Debra McNamara and Jan Ipenburg, Rheumatology Clinical Nurse Specialists, and Orthopaedic Surgeons Alastair Rothwell, Andrew Vincent, and Steve Parkinson in Christchurch and Michael Chin, John Matheson, and David Gywnne-Jones in Dunedin for assistance in obtaining synovial samples.

\section{REFERENCES}

1. Kirkham BW, Lassere M, Edmonds J, Juhasz K, Bird P, Lee C, et al. Synovial membrane cytokine expression is predictive of joint damage progression in rheumatoid arthritis. Arthritis Rheum 2006;54:1122-31.

Personal non-commercial use only. The Journal of Rheumatology Copyright @ 2009 . All rights reserved. 
2. Chabaud M, Durand JM, Buchs N, Fossiez F, Page G, Frappart L, et al. Human interleukin-17: A T cell-derived proinflammatory cytokine produced by the rheumatoid synovium. Arthritis Rheum 1999;42:963-70.

3. Bettelli E, Carrier Y, Gao W, Korn T, Storm T, Oukka M, et al. Reciprocal developmental pathways for the generation of pathogenic effector TH17 and regulatory T cells. Nature 2006;441:235-8.

4. Harrington L, Hatton R, Mangan P, Turner H, Murphy T, Murphy $\mathrm{K}$, et al. Interleukin 17-producing CD4+ effector T cells develop via a lineage distinct from the $\mathrm{T}$ helper type 1 and 2 lineages. Nature Immunol 2005;6:1123-32.

5. Chen Z, Tato C, Muul C, Laurence A, O'Shea J. Distinct regulation of interleukin-17 in human T helper lymphocytes. Arthritis Rheum 2007;56:2936-46

6. Hoeve M, Savage N, de Boer T, Langenberg D, Malefyt R, Ottenhoff T, et al. Divergent effects of IL-12 and IL-23 on the production of IL-17 by human T cells. Eur J Immunol 2006:36:661-70.

7. Steinman L. The rush to judgement on Th17. J Exp Med 2008;205:1517-22.

8. Arnett FC, Edworthy SM, Bloch DA, McShane DJ, Fries JF, Cooper NS, et al. The American Rheumatism Association 1987 revised criteria for the classification of rheumatoid arthritis. Arthritis Rheum 1988;31:315-24.

9. Stamp L, Easson A, Lehnigk U, Highton J, Hessian P. Different T cell subsets in the nodule and synovial membrane - absence of IL-17A in rheumatoid nodules. Arthritis Rheum 2008;58:1601-8.

10. Singh R, Aggarwal A, Misra R. Th1/Th17 cytokine profiles in patients with reactive arthritis/undifferentiaed spondyloarthropathy. J Rheumatol 2007;34:2285-90.

11. Kohno M, Tsutsumi A, Matsui H, Sugihara M, Suzuki T, Mamura $\mathrm{M}$, et al. Interleukin-17 gene expression in patients with rheumatoid arthritis. Mod Rheumatol 2008;18:15-22.

12. Kim H-R, Cho M-L, Kim K-W, Juhn J-Y, Hwang S-Y, Yoon C-H, et al. Up-regulation of IL-23p19 expression in rheumatoid arthritis synovial fibroblasts by IL-17 through PI3-kinase-, NF-kB- and p38 MAPK-dependent signalling pathways. Rheumatology 2007;46:57-64
13. Kim H-R, Kim H-S, Park M-K, Cho M-L, Lee S-H, Kim H-Y. The clinical role of IL-23p19 in patients with rheumatoid arthritis. Scand J Rheumatol 2007;36:259-64.

14. Kageyama Y, Ichikawa T, Nagafusa T, Torikai E, Shimazu M, Nagano A. Etanercept reduces the serum levels of interleukin-23 and macrophage inflammatory protein- 3 alpha in patients with rheumatoid arthritis. Rheumatol Int 2007;28:137-43.

15. Oppman B, Lesley R, Blom B, Timans Y, Xu Y, Hunte B, et al Novel p19 protein engages IL-12p40 to form a cytokine, IL-23, with biological activities similar as well as distinct from IL-12. Immunity 2000;13:715-25.

16. Brentano F, Ospelt C, Stanczyk J, Gay R, Gay S, Kyburz D. Abundant expression of the IL-23 subunit p19, but low levels of bioactive IL-23 in the rheumatoid synovium [abstract]. Ann Rheum Dis 2009;68:143-50.

17. Deng J, Younge B, Goronzy J, Weyand C. Th17 cells as mediators of vessel wall damage in giant cell arteritis. Arthritis Rheum 2008; Suppl 58:S392.

18. Yamada H, Nakashima Y, Okazaki K, Mawatari T, Fukuchi J-I, Kaibara N, et al. Th1 but not Th17 cells predominate in the joints of patients with rheumatoid arthritis. Ann Rheum Dis 2008;67:1299-304. Epub 2007 Dec 6.

19. Yoshimura T, Takeda A, Hamano S, Miyazaki Y, Kinjyo I, Ishibashi $\mathrm{T}$, et al. Two-sided roles of IL-27: Induction of Th1 differentiation on naive $\mathrm{CD} 4+\mathrm{T}$ cells versus suppression of proinflammatory cytokine production including IL-23-induced IL-17 on activated CD4+ T cells partially through STAT3-dependent mechanism. J Immunol 2006;177:5377-85.

20. Raza K, Falciani F, Curnow S, Ross E, Lee C-Y, Akbar A, et al. Early rheumatoid arthritis is characterised by a distinct and transient synovial fluid cytokine profile of T cell and stromal cell origin. Arthritis Res Ther 2005;7:R784-95. 